\title{
COMMENTARY
}

\section{The liver in sepsis: shedding light on the cellular basis of hepatocyte dysfunction}

\author{
John C Marshall* \\ See related research by Gonnert et al., http://ccforum.com/content/17/2/R67
}

\begin{abstract}
Liver dysfunction is believed to contribute to the metabolic derangements of critical illness. The cellular basis of liver dysfunction is poorly understood and its consequences largely unknown. Recent work by Gonnert and colleagues sheds additional light. Using two imaging techniques to track the clearance of biotransformed dyes by the liver in a rat model of intra-abdominal infection, the authors show that the predominant defect in sepsis lies in the excretion of biotransformed molecules from the hepatocyte into the bile canaliculi. Their work both points to a new aspect of hepatic dysfunction through focus on a role in the metabolic derangements of sepsis and suggests a possible strategy to diagnose and monitor this process in critically ill patients.
\end{abstract}

A new report by Gonnert and co-workers from the Center for Sepsis Control and Care in Jena Germany, recently appearing in Critical Care, provides important new insight into the nature and mechanisms of hepatocellular dysfunction in sepsis [1]. Since Bywaters first described the phenomenon of jaundice following severe trauma in 1946 [2], liver dysfunction has been considered a prominent feature of the Multiple Organ Dysfunction Syndrome and has been defined predominantly by hyperbilirubinemia and clinical jaundice [3-6]. The liver, however, is an enormously complex organ whose function is difficult to characterize succinctly or comprehensively. The liver plays a critical role in protein synthesis, in intermediary metabolism, and in the excretion of toxins and wastes, including the breakdown products of senescent red cells; this latter role is reflected in the widespread use of bilirubin levels as a measure of hepatic dysfunction.

*Correspondence: marshallj@smh.ca

St Michael's Hospital, $4^{\text {th }}$ Floor Bond Wing, Room 4-007, 30 Bond Street, Toronto, Ontario, Canada M5B 1W8
However, the liver is also involved in hematopoiesis during embryologic development, in innate immunity, and in digestion through the production of bile.

Yet we understand little about how liver function is altered during critical illness, and how these alterations are best measured. Hyperbilirubinemia, the classic marker of hepatic dysfunction, provides little insight into what is happening within the liver. The prevalence of hyperbilirubinemia appears to be on the decline, perhaps because of declining use of total parenteral nutrition and of transfusion, although it is an independent risk factor for increased mortality $[7,8]$. Moreover, the presence and prognostic import of hyperbilirubinemia appear to derive more from the fact that this marker delineates a population of patients with irreversible end-stage liver disease rather than a transient and potentially reversible process that might be considered a component of the Multiple Organ Dysfunction Syndrome. Greater insight into other aspects of hepatic dysfunction that might be both deranged in critical illness and measured in critically ill patients is clearly needed.

The Jena group is making substantial strides in addressing this need, and is bringing attention to striking abnormalities in hepatic biotransformation and excretion that arise in severely ill patients [9]. They have shown previously that hepatocellular transport at the canalicular pole of the hepatocyte is impaired in sepsis, with the result that a spectrum of hepatotoxic materials accumulates within the septic liver. They now extend these important observations [1].

Gonnert and colleagues use a combination of elegant imaging techniques to detect clearance of two dyes that are metabolized by the liver - indocyanine green and DY635 - in a lethal rat model of intra-abdominal infection resulting from intraperitoneal administration of feces. They show that dye is cleared from the circulation and taken up readily by liver cells, but that its appearance in bile is delayed, indicating that defective cellular function primarily involves excretion of transformed molecules from the hepatocyte into the biliary canaliculi, rather than its delivery to, or uptake by, liver cells. Impaired excretion of dye is further evidenced as 
sustained persistence of dye within the liver, and its reduced appearance within the lumen of the gastrointestinal tract.

This body of work and the ongoing evolution of the authors' investigations hold exciting and important possibilities. First, the work provides valuable insight into the nature of hepatocellular dysfunction in critical illness, shifting the focus from microcirculatory impairments or abnormalities in uptake of hepatically transformed compounds to their excretion into the biliary tree and gastrointestinal tract. The significance of accumulation of a spectrum of compounds - toxins, drugs, bilirubin, and other metabolic substances - within the hepatocyte is uncertain, and is fertile ground for future study. Does their accumulation subsequently result in impaired uptake from the liver microvasculature? If so, then toxins or drugs may accumulate in the circulation, their normal metabolism impeded by failure of their excretion from the hepatocyte. If this is the case, then those alterations may be modifiable by altering drug doses or by extracorporeal removal, and eventually by pharmacologic interventions that correct the excretory derangement. Does impaired hepatocellular excretion affect the normal synthetic function of the hepatocyte, and therefore impair its ability to synthesize proteins such as acute phase reactants or coagulation factors? Does impaired excretion alter the ability of the liver to take up glucose and store it as glycogen, and therefore may hepatocellular dysfunction emerge as a component of the syndrome of insulin resistance that is so commonly seen in sepsis? Do alterations in hepatocyte function result in altered Kupffer cell function, and therefore contribute to the immunologic alterations of sepsis?

Moreover, as techniques of near-infrared fluorescence imaging improve, the technologies described by Gonnert and colleagues may well find a clinical role as a noninvasive test of hepatic dysfunction in the critically ill patient, and so provide a measure of liver function that is more sensitive, sophisticated, and relevant than the serum bilirubin level.

Despite its fundamental and varied role in normal homeostasis, to the intensivist the liver remains a frustratingly opaque black box whose inner workings are obscure. Studies like those of Gonnert and colleagues are beginning to lift the lid, if only a little, and to shine a light on a largely unexplored area in the support of the critically ill patient.

\section{Competing interests}

JCM serves as an unpaid member of the Advisory Board for the Center for Sepsis Control and Care of the University of Jena and receives support for travel and accommodation to attend these meetings.

\section{Acknowledgements}

This work was supported in part by grants from the Canadian Institutes for Health Research and the Physicians Services Incorporated Foundation.

Published: 12 June 2013

\section{References}

1. Gonnert FA, Recknagel P, Hilger I, Claus RA, Bauer M, Kortgen A: Hepatic excretory function in sepsis: implications from biophotonic analysis of transcellular xenobiotic transport in a rodent model. Crit Care 2013, 17:R67.

2. Bywaters EGL: Anatomical changes in the liver after trauma. Clin Sci 1946, 6:19.

3. Skillman JJ, Bushnell LS, Goldman H, Silen W: Respiratory failure, hypotension, sepsis, and jaundice. A clinical syndrome associated with lethal hemorrhage and acute stress ulceration in the stomach. Am J Surg 1969, 117:523-530

4. Fry DE, Pearlstein L, Fulton RL, Polk HC: Multiple system organ failure. The role of uncontrolled infection. Arch Surg 1980, 115:136-140.

5. Marshall JC, Cook DJ, Christou NV, Bernard GR, Sprung CL, Sibbald WJ: Multiple organ dysfunction score: a reliable descriptor of a complex clinical outcome. Crit Care Med 1995, 23:1638-1652.

6. Vincent J-L, Moreno R, Takala J, Willatts S, De Mendonça A, Bruining H, Reinhart CK, Suter PM, Thijs LG: The SOFA (Sepsis-related Organ Failure Assessment) score to describe organ dysfunction/failure. On behalf of the Working Group on Sepsis-related Problems of the European Society of Intensive Care Medicine. Intensive Care Med 1996, 22:707-710.

7. Harbrecht BG, Zenati MS, Doyle HR, McMichael J, Townsend RN, Clancy KD, Peitzman AB: Hepatic dysfunction increases length of stay and risk of death after injury. J Trauma 2002, 53:517-523.

8. Kramer $L$, Jordan B, Druml W, Bauer P, Metnitz PG: Incidence and prognosis of early hepatic dysfunction in critically ill patients - a prospective multicenter study. Crit Care Med 2007, 35:1099-1104.

9. Recknagel P, Gonnert FA, Westermann M, Lambeck S, Lupp A, Rudiger A, Dyson A, Carré JE, Kortgen A, Krafft C, Popp J, Sponholz C, Fuhrmann V, Hilger I, Claus RA, Riedemann NC, Wetzker R, Singer M,Trauner M, Bauer M: Liver dysfunction and phosphatidylinositol-3-kinase signalling in early sepsis: experimental studies in rodent models of peritonitis. PLOS Med 2012, 9:e1001338.

doi:10.1186/cc12731

Cite this article as: Marshall JC: The liver in sepsis: shedding light on the cellular basis of hepatocyte dysfunction. Critical Care 2013, 17:153. 